\title{
Endoscopic management of pharyngeal pouch with difficult access using Harmonic Scalpell: How we do it R Anmolsingha,b, K Lee ${ }^{a, b}, C^{a b o}{ }^{a}$ \\ a Department of ENT, Royal Bolton NHS Trust b ENT Specialty Registrar, Health Education North West, Englandc
}

Objective:To describe the transoral treatment of Pharyngeal Pouch with a harmonic scalpel and review the initial outcomes of patients treated with this technique.

Method:A retrospective study of 6 patient case series over the period 2014-2017 who underwent endoscopic repair of pharyngeal pouch by harmonic scalpel. Electronic medical records were reviewed. Extracted data included outpatient clinic notes, operative reports, hospital summaries, and follow-up visits.

Surgical technique: After the induction of general anesthesia, oesophagoscopy was performed using a rigid oesophagoscope exposing the common wall between the oesophagus and the pouch. Although adequate exposure was obtained in each case, successful stapling could not be performed because of either shallow diverticulum depth or trismus preventing proper insertion of the stapling device. Harmonic scalpel (Harmonic Ace, Ethicon Endo-Surgery, Inc, Cincinnati, OH) was used to divide the common wall between the diverticulum and oesophagus, sealing the cut mucosal edges and performing a cricopharyngeal myotomy in the process. The serosa was then inspected to ensure there was no breach.All patients were discharged on the same day of the procedure. Symptom improvement was assessed during subsequent follow-up

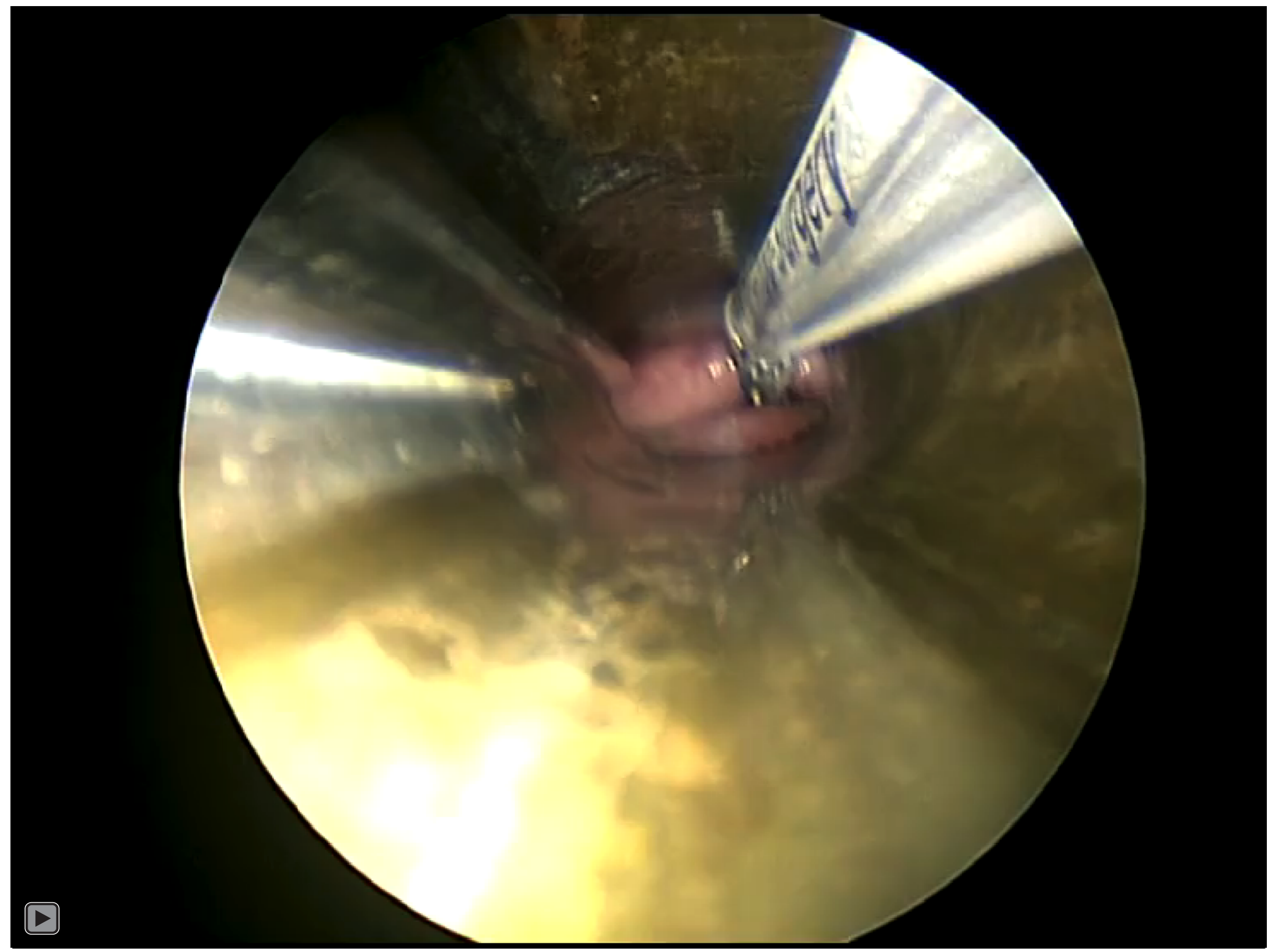

Video I (click video to play): Insertion of rigid oesophagoscope and visualization, using 0 degree rigid endoscope, of wall between pharyngeal pouch and oesophagus. Harmonic Scalpel then inserted and used to dissect the mucosa and perform cricopharyngeal myotomy.

Results: 5 patients were treated successfully and safely with the harmonic scalpel. I patient had recurrence of the pharyngeal pouch symptoms 3 years post op and is currently awaiting revision surgery. None of the patients experienced post op haemorrhage, mediastinitis, subcutaneous emphysema.

Discussion: In the above described patients, satisfactory repair could not be performed endoscopically using the stapling device because of shallow diverticulum depth and anatomical factors preventing the introduction of the stapler into the diverticulum. Instead, the harmonic scalpel was used. The smaller diameter of the harmonic scalpel allows it to be manoeuvred and positioned in cases where the larger stapling device will not fit.

Conclusion: Harmonic scalpel use in endoscope repair of pharyngeal pouch is a safe and efficacious procedure that allows for treatment in certain cases that cannot be treated by endoscopic stapling devices. Further study is warranted in larger series to determine efficacy and safety compared with established endoscopic techniques 